Original Article

\title{
Obstructive sleep apnea may induce orexinergic system and cerebral $\beta$-amyloid metabolism dysregulation: is it a further proof for Alzheimer's disease risk?
}

\author{
Claudio Liguori ${ }^{\text {a, * }}$, Nicola Biagio Mercuri ${ }^{\text {a, b, c }}$, Marzia Nuccetelli ${ }^{\text {d }}$, Francesca Izzi ${ }^{\text {a }}$, \\ Alberto Cordella ${ }^{\mathrm{b}}$, Sergio Bernardini ${ }^{\mathrm{d}}$, Fabio Placidi ${ }^{\mathrm{a}}$ \\ a Sleep Medicine Centre, Neurophysiopathology Unit, Department of Systems Medicine, University of Rome "Tor Vergata", Rome, Italy \\ ${ }^{\mathrm{b}}$ Fondazione Santa Lucia IRCCS, Rome, Italy

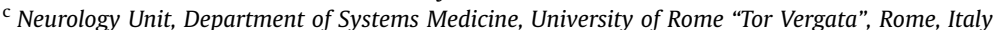 \\ d Department of Clinical Biochemistry and Molecular Biology, University of Rome "Tor Vergata", Rome, Italy
}

\section{A R T I C L E I N F O}

\section{Article history:}

Received 13 October 2018

Received in revised form

1 December 2018

Accepted 2 January 2019

Available online 11 January 2019

\section{Keywords:}

Obstructive sleep apnea

$\beta$-Amyloid

Alzheimer's disease

Orexin

Sleep

\begin{abstract}
A B S T R A C T
Background: Obstructive Sleep Apnea (OSA) is associated with pathological changes of cerebral $\beta$-amyloid dynamics. Orexin has been demonstrated interfering with $\beta$-amyloid metabolism in Alzheimer's Disease $(A D)$ pathology. The present study investigated cerebrospinal-fluid (CSF) $\beta$-amyloid $40\left(A \beta_{40}\right), \beta$ amyloid $_{42}\left(A \beta_{42}\right)$ and orexin levels in OSA patients compared to AD patients and controls.

Methods: OSA and AD patients were included in this study and compared to a group of controls. Patients and controls underwent lumbar puncture for the assessment of CSF A $\beta_{40}, A \beta_{42}$, tau proteins, orexin levels, and polysomnography to measure nocturnal sleep architecture.

Results: 20 OSA patients, 20 AD patients, and 15 controls were included in our study. OSA patients showed higher CSF orexin levels than AD patients and controls, and AD patients showed higher CSF orexin levels than controls. Moreover, CSF $A \beta_{40}$ and $A \beta_{42}$ were lower in OSA patients than controls, but higher in OSA patients compared to AD patients. However, AD patients showed lower CSF A $\beta_{42}$ levels but comparable CSF $A \beta_{40}$ levels than controls. Sleep macrostructure was similarly altered in OSA and $A D$ patients compared to controls. Finally, the apnea-hypopnea index (AHI) was related to the ratio $A \beta_{42} /$ $\mathrm{A} \beta_{40}$ and CSF orexin levels in OSA patients.

Conclusion: This study proved the alteration of CSF orexin levels and $\beta$-amyloid isoforms 40 and 42 in OSA patients. We suppose that sleep disruption and intermittent hypoxia, the two core features of OSA, may induce orexinergic system and cerebral $\beta$-amyloid metabolism dysregulation. This evidence further supports the current hypothesis that OSA may possibly start AD neuropathological processes.
\end{abstract}

(c) 2019 Elsevier B.V. All rights reserved.

\section{Introduction}

Sleep impairment may affect cerebral function and recent findings documented that sleep participates in the clearance of brain metabolic products accumulating during wakefulness [1]. Among cerebral catabolic products, $\beta$-amyloid is cleared from the brain interstitial space during sleep [1]. It has been hypothesized

\footnotetext{
* Corresponding author. Sleep Medicine Centre Neurophysiopathology Unit, Department of Systems Medicine University of Rome "Tor Vergata”, Viale Oxford 81, 00133, Rome, Italy. Fax: +390620902116.

E-mail address: dott.claudioliguori@yahoo.it (C. Liguori).
}

that following cerebral aggregation and deposition of $\beta$-amyloid 42 $\left(A \beta_{42}\right)$ Alzheimer's Disease (AD) neurodegenerative processes may start [2]. Not only $A \beta_{42}$ but also $\beta$-amyloid an $_{40}\left(A \beta_{40}\right)$ is a catabolic product of the amyloid-precursor protein [3]. Reduction of cerebrospinal-fluid (CSF) $A \beta_{40}$ and $A \beta_{42}$ levels has been recently documented in cross sectional studies comparing patients affected by obstructive sleep apnea (OSA) to controls [4,5]. It has been suggested that sleep impairment due to OSA condition may induce preclinical AD biomarkers changes, as the reduction of CSF $A \beta_{42}$ levels and the increase of brain amyloid plaques deposition [4-8]. Accordingly, OSA has been identified as a possible risk factor for dementia and AD [9]. However, although the field of sleep and neurodegeneration is still young, these findings raised attention 


\begin{tabular}{|c|c|c|c|}
\hline \multicolumn{2}{|c|}{ List of abbreviations } & \multirow{2}{*}{$\begin{array}{l}(\text { OSA }) \\
(\mathrm{p}-\mathrm{tau}) \\
(\mathrm{PSG})\end{array}$} & \multirow{2}{*}{$\begin{array}{l}\text { obstructive sleep apnea } \\
\text { phosphorylated-tau } \\
\text { Polysomnography }\end{array}$} \\
\hline$\left(A \beta_{40}\right)$ & $\beta$-amyloid 40 & & \\
\hline$\left(A \beta_{42}\right)$ & $\beta$-amyloid 42 & (REM) & REM sleep \\
\hline$(\mathrm{AD})$ & Alzheimer's Disease & (LREM) & REM sleep latency \\
\hline (AASM) & American Academy of Sleep Medicine & (SE) & sleep efficiency \\
\hline$(\mathrm{AHI})$ & Apnea-Hypopnea Index & (N1) & stage 1 of non-REM sleep \\
\hline (BMI) & body mass index & $(\mathrm{N} 2)$ & stage 2 of non-REM sleep \\
\hline$(\mathrm{CSF})$ & cerebrospinal fluid & (N3) & stage 3 of non-REM sleep \\
\hline (ESS) & Epworth Sleepiness Scale & (TST) & total sleep time \\
\hline$(\mathrm{IH})$ & intermittent hypoxia & (t-tau) & total tau \\
\hline$(\mathrm{LP})$ & lumbar puncture & (WASO) & wakefulness after sleep onset \\
\hline (MMSE) & Mini Mental State Examination & & \\
\hline
\end{tabular}

about the possible association between OSA and AD, considering that OSA is a worldwide increasingly common disorder. Hence, further evidence about this association is currently invoked.

OSA is characterized by the occurrence of repetitive episodes of upper airways obstruction leading to sleep fragmentation and intermittent hypoxia (IH) [10]. Severity of OSA is determined by the sum of all the apneas and hypopneas per hour of sleep, named Apnea-Hypopnea Index (AHI) [11]. Both sleep fragmentation and IH may affect brain structure and function, alter the cerebral $\beta$-amyloid dynamics and lower $\beta$-amyloid clearance, thus increasing its production and reducing its metabolism [4-6,12-14].

Orexin-A (Hypocretin-1) is a neuropeptide produced by the lateral hypothalamic neurons, and regulates the sleep-wake cycle by increasing and maintaining wakefulness [15]. The orexinergic system is altered in AD pathology and its dysregulation has been related to sleep impairment in $\mathrm{AD}$ patients from the early stages of the disease [16-19]. Moreover, orexin has been demonstrated influencing $\beta$-amyloid metabolism in animal models studies [20]. In the past years, exclusively plasma orexin levels have been measured in OSA patients, and only one study assessed CSF orexin levels in mild OSA patients [21-23].

Based on the previous observation from our group documenting lower CSF $A \beta_{42}$ levels in OSA patients [4], in this study we aimed to assess CSF $\beta$-amyloid biomarkers $\left(A \beta_{42}, A \beta_{40}\right)$ and orexin levels in order to evaluate the possible relationship between brain $\beta$-amyloid levels, orexinergic system and sleep impairment in OSA patients. Hence, we investigate in OSA patients compared to $A D$ patients and controls: (i) CSF AD biomarkers ( $\beta$-amyloid and tau proteins) and orexin levels; (ii) sleep architecture measured via polysomnography (PSG); and (iii) the possible relationships among nocturnal sleep, CSF AD biomarkers and orexin in OSA and AD groups.

\section{Methods}

\subsection{Participants and study design}

OSA $(A H I \geq 15 / h)$ patients, $A D$ patients and controls were consecutively included in this study between October 2015 and June 2018. This study followed a previous observation documenting altered CSF AD biomarkers in patients affected by OSA [4]. All patients included in the present study underwent a standard screening at the Neurology Clinic of the University Hospital of Rome "Tor Vergata", including physical and neurological examinations, Mini Mental State Examination (MMSE), laboratory tests, PSG, brain magnetic resonance imaging (MRI) and lumbar puncture (LP) for CSF biomarkers analysis, including $A \beta_{42}, A \beta_{40}$, phosphorylated-tau ( $\mathrm{p}$-tau), total tau (t-tau), and orexin levels. In particular, in this study, unlike our previous study [4], the primary aim of this study was to measure $C S F A \beta_{42}, A \beta_{40}$, and orexin levels in moderate to severe OSA patients compared to both AD patients and controls.

$\mathrm{AD}$ diagnosis was based on the currently proposed biomarkers diagnostic criteria for $\mathrm{AD}$, which was based on the clinical recognition of dementia associated with biomarkers consistent with $A D$ pathology. This implies the pathological reduction of CSF levels of $A \beta_{42}$ coupled with pathological high CSF levels of t-tau and $p$-tau, medial temporal lobe atrophy on MRI, and cortical temporoparietal hypometabolism on [18F]FDG positron emission tomography [13].

OSA was determined by nocturnal polysomnographic recording, performed according to American Academy of Sleep Medicine (AASM) criteria [24]. We defined apnea as the reduction $>90 \%$ of respiratory airflow and hypopnea as the reduction $>30 \%$ of respiratory airflow associated with an oxygen desaturation $\geq 3 \%$. Both apnea and hypopnea should continue for 10 or more seconds [24]. Severity of OSA was determined by the frequency of apneas and hypopneas, which constitutes the AHI.

$\mathrm{OSA}$ and $\mathrm{AD}$ patients required to meet the following entry criteria for this study: no concomitant neurologic or psychiatric diseases; no intake of drugs active on the CNS; no use of caffeine, tobacco and/or alcohol at the time of the sleep investigations. Exclusion criteria were as follows: abnormal cell count ( $>4$ white cells/mcL) at the CSF sample analysis; subclinical seizures and chronic obstructive pulmonary disease; and previous diagnosis of sleep disorders based on AASM criteria other than OSA. Moreover, OSA patients underwent a comprehensive neuropsychological testing in order to exclude mild cognitive impairment.

We also included in this observation a control group constituted by patients admitted for suspected polyneuropathy from October 2015 to June 2018, ruled out after diagnostic investigations. All patients underwent clinical neurological investigation, brain MRI, PSG and LP at the same Neurology Clinic. Specific exclusion criteria for controls were as follows: intake of CNS active drugs; intake of melatonin supplements or hypnotics; consumption of caffeine, tobacco and/or alcohol at the time of the sleep laboratory investigation; and previous diagnosis of other neurological and/or psychiatric diseases. Moreover, since the high prevalence of mild OSA in $\mathrm{AD}$ patients and adult-elderly controls based on the current literature, we set the AHI cut-off at $15 / \mathrm{h}$ for both groups $[25,26]$. Notably, we excluded AD patients and controls who presented OSA syndrome defined according to AASM guidelines [24].

Finally, the Epworth Sleepiness Scale (ESS), a wide used instrument to assess daytime sleepiness, was administered to $A D$, OSA and controls [27,28].

Therefore, we compared MMSE, CSF and PSG data among AD, OSA, and control groups. 


\subsection{Polysomnographic recording}

Patients underwent PSG in order to evaluate their nocturnal sleep (SOMNOscreen, SOMNOmedics GmbH, Randersacker, Germany), as already reported [17]. The following standard parameters were analyzed: total sleep time (TST), sleep efficiency (SE), wakefulness after sleep onset (WASO), stage 1 of non-REM sleep (N1), stage 2 of non-REM sleep (N2), stage 3 of non-REM sleep (N3), REM sleep (REM), and REM sleep latency (LREM). Sleep stages percentages were calculated during the TST [24]. AHI was defined as mentioned above and used for OSA diagnosis [24]. Blinded researchers (CL, FP, FI) scored PSG recordings on the basis of the international standard criteria of the American Academy of Sleep Medicine [24].

\subsection{CSF collection and analysis}

All CSF samples were obtained the day after the PSG recording by LP performed in the decubitus position between 8:00 and 9:30 $\mathrm{AM}$, within 1-2 $\mathrm{h}$ after morning awakening, using an atraumatic needle. CSF collection was performed as previously reported [17-19]. CSF $A \beta_{40}, A \beta_{42}$, t-tau and $p$-tau levels were determined according to previously published standard procedures, using commercially available sandwich enzyme-linked immunosorbent assays (Innotest $\beta$-Amyloid 1-40, Innotest $\beta$-Amyloid 1-42, Innotest h-T-tau, Innotest Phospho-T-tau 181; Innogenetics, Ghent, Belgium) [29,32]. CSF orexin levels were detected according to previously published standard procedures with commercially available radioimmunoassay kit (Orexin A/Hypocretin-1 RIA Kit; Phoenix Pharmaceuticals, Burlingame, CA) [33]. The two researchers (NM, BS) who performed the analysis were blinded to the clinical status of participants.

$A \beta_{42}$, t-tau, and p-tau were dichotomized on the basis of previously established literature-based and in-laboratory confirmed cut-off values: $<500 \mathrm{pg} / \mathrm{mL}$ for $A \beta_{42},>375 \mathrm{pg} / \mathrm{mL}$ for t-tau, and $>52 \mathrm{pg} / \mathrm{mL}$ for $\mathrm{p}$-tau [27-30]. The t-tau/A $\beta_{42}$ and $A \beta_{42} / A \beta_{40}$ ratios were also calculated, and the cut-off $>0.52$ and $<0.06$ were considered to represent the CSF profile suggestive of AD [14,29].

\subsection{Data and statistical analysis}

The study protocol was considered as observational by the Independent Ethical Committee of the University Hospital of Rome "Tor Vergata" and was then performed according to the STROBE statement. Patients and controls provided their informed consent to the study.

Statistical analysis was performed by using the commercial software Statistica 10.0 program; Statsoft Inc, Tulsa, OK, USA.

The Kolmogorov-Smirnov test was used to check for normal distribution of the obtained PSG and CSF data. All demographical, clinical, PSG, and CSF data were then compared among the three groups (OSA vs AD vs controls) using the one-way analysis of variance, body mass index (BMI), age and sex as covariates. The post-hoc analysis was performed using the Tukey test for honestly significant difference. P value $<0.05$ was considered to be statistically significant. Pearson correlation test was used to separately correlate CSF biomarkers levels and PSG data in OSA, AD and control groups. An additional multiple regression analysis was performed. Data were analyzed with a significant $\mathrm{p}$ value of 0.05 .

\section{Results}

\subsection{Demographic and clinical data}

We included in this study 20 OSA patients, 20 AD patients and 15 controls. Demographic and clinical data are represented in Table 1.
OSA patients were younger and more frequently male than AD patients and showed a higher BMI than both AD patients and controls. MMSE was similar in OSA patients and controls, who showed higher scores compared to AD patients (Table 1). Moreover, the three groups did not differ in terms of frequency of apoE4 genotype (Table 1).

\subsection{CSF data}

Comparing CSF data, we found that AD patients showed lower CSF $A \beta_{42}$ levels as well as higher t-tau and p-tau CSF levels with respect to both OSA and controls (see Table 1 ). Significantly, OSA patients showed lower CSF $A \beta_{42}$ levels than controls (see Table 1 ). In particular, 9/20 OSA patients showed pathologically low CSF A $\beta_{42}$ levels $(<500 \mathrm{pg} / \mathrm{mL})$. Conversely, CSF levels of t-tau and p-tau did not differ between OSA and control groups (see Table 1). T-tau/A $\beta_{42}$ ratio was higher in $A D$ compared to OSA patients and controls, and $A \beta_{42} / A \beta_{40}$ ratio was lower in $A D$ compared to OSA patients and controls (see Table 1 ). OSA patients showed higher T-tau/A $\beta_{42}$ ratio and lower $A \beta_{42} / A \beta_{40}$ ratio than controls (see Table 1 ). Notably, OSA patients showed lower CSF $A \beta_{40}$ levels compared to both $A D$ patients and controls (see Table 1).

Considering CSF orexin levels, we found higher CSF orexin concentrations in OSA patients compared to both AD patients and controls. Furthermore, AD patients showed higher CSF orexin levels compared to controls (see Table 1).

All CSF data of AD, OSA and controls are reported in Table 1.

\subsection{PSG data}

Comparing PSG parameters among AD, OSA, and control groups, we found reduced $S E$ in $O S A$ and $A D$ patients with respect to controls (see Table 2). AD patients showed lower TST than both OSA and controls, but OSA patients showed an higher TST than controls. Analyzing the macro-structural sleep parameters, we documented similar alterations in OSA and AD patients, since both groups showed higher N1 and N2 and lower N3 compared to controls (see Table 2). Moreover, AD patients showed lower N3 compared to OSA patients. LREM was higher and REM was lower in AD and OSA patients compared to controls (see Table 2). Finally, higher WASO was documented in AD and OSA patients than controls (see Table 2). As expected, OSA patients showed higher AHI compared to AD and controls.

All PSG data are reported in Table 2.

\subsection{Correlations between CSF and PSG data}

Considering OSA group we firstly correlated CSF data and documented the significant correlation between t-tau $/ A \beta_{42}$ ratio and as well as orexin CSF levels $(\mathrm{R}=-0.52, \mathrm{p}<0.01)$, and between CSF $A \beta_{40}$ levels and as well as both t-tau and p-tau CSF levels $(\mathrm{R}=0.57, \mathrm{p}<0.01$ and $\mathrm{R}=0.61 \mathrm{p}<0.01)$. Moreover, considering CSF and PSG data in the OSA group and documented the significant correlation between CSF $A \beta_{40}$ levels and both WASO $(R=0.51$, $\mathrm{p}<0.01)$ and $\mathrm{SE}(\mathrm{R}=-0.53, \mathrm{p}<0.01)$, and between $\mathrm{A} \beta_{42} / \mathrm{A} \beta_{40}$ ratio and $\mathrm{AHI}(\mathrm{R}=-0.46, \mathrm{p}<0.01$; see Fig. 1$)$.

Considering CSF data in AD group, we found that CSF orexin levels significantly correlated with CSF $A \beta_{40}$ levels $(R=0.67$, $\mathrm{p}<0.01), A \beta_{42} / \mathrm{A} \beta_{40}$ ratio $(\mathrm{R}=-0.72, \mathrm{p}<0.01), \mathrm{t}-\mathrm{tau} / \mathrm{A} \beta_{42}$ ratio $(\mathrm{R}=0.81, \mathrm{p}<0.01)$, CSF t-tau $(\mathrm{R}=0.87, \mathrm{p}<0.01)$ and $\mathrm{p}$-tau $(\mathrm{R}=0.84, \mathrm{p}<0.01)$ levels. Moreover, $A \beta_{42} / A \beta_{40}$ ratio correlated with CSF t-tau and p-tau levels $(\mathrm{R}=-0.71, \mathrm{p}<0.01$ and $\mathrm{R}=-0.76$, $\mathrm{p}<0.01$ ). CSF $A \beta_{40}$ levels correlated with CSF t-tau and $\mathrm{p}$-tau levels $(\mathrm{R}=0.61, \mathrm{p}<0.01$ and $\mathrm{R}=0.83, \mathrm{p}<0.01)$. Correlating CSF and PSG data in the $\mathrm{AD}$ group, we documented the significant correlation between CSF orexin levels and both REM and N3 $(\mathrm{R}=-0.57$, $\mathrm{p}<0.01$ and $\mathrm{R}=-0.62, \mathrm{p}<0.01$ ). 
Table 1

Clinic, Demographic and CSF data of OSA, AD, and Control groups.

\begin{tabular}{|c|c|c|c|c|}
\hline & $\begin{array}{l}\text { OSA }(\mathrm{n}=20) \\
(\text { mean } \pm S D)\end{array}$ & $\begin{array}{l}\mathrm{AD}(\mathrm{n}=20) \\
(\text { mean } \pm S D)\end{array}$ & $\begin{array}{l}\text { Controls }(\mathrm{n}=15) \\
(\text { mean } \pm S D)\end{array}$ & P Value \\
\hline Age & $58.75 \pm 3.53$ & $66.3 \pm 4.18$ & $63.8 \pm 8.46$ & $\mathrm{OSA}<\mathrm{AD}, \mathrm{p}<0.05$ \\
\hline Sex & $14 \mathrm{M} 6 \mathrm{~F}$ & 7M13F & $8 \mathrm{M} 7 \mathrm{~F}$ & OSA vs $\mathrm{AD}, \mathrm{p}<0.01$ \\
\hline BMI & $27.63 \pm 2.65$ & $24.41 \pm 1.97$ & $24.59 \pm 1.89$ & $\begin{array}{l}\text { OSA vs AD, } \mathrm{p}<0.05 \\
\text { OSA vs Controls, } \mathrm{p}<0.05\end{array}$ \\
\hline MMSE & $29.1 \pm 1.07$ & $21.4 \pm 1.93$ & $29.6 \pm 1.47$ & $\begin{array}{l}\text { AD vs OSA, } \mathrm{p}<0.01 \\
\mathrm{AD} \text { vs Controls, } \mathrm{p}<0.01\end{array}$ \\
\hline ESS & $13.5 \pm 3.68$ & $6.7 \pm 3.87$ & $5.11 \pm 3.76$ & $\begin{array}{l}\text { OSA vs AD, } \mathrm{p}<0.01 \\
\text { OSA vs Controls, } \mathrm{p}<0.01\end{array}$ \\
\hline apoE4 & $8 / 20$ & $11 / 20$ & $6 / 15$ & NS \\
\hline $\mathrm{A} \beta 40(\mathrm{pg} / \mathrm{mL})$ & $7090.45 \pm 2739.1$ & $11652.8 \pm 5388.41$ & $10561.6 \pm 2688.47$ & $\begin{array}{l}\text { OSA vs AD, } \mathrm{p}<0.01 \\
\text { OSA vs Controls, } \mathrm{p}<0.05\end{array}$ \\
\hline $\mathrm{A} \beta 42(\mathrm{pg} / \mathrm{mL})$ & $622.05 \pm 270.43$ & $327.4 \pm 132.6$ & $889.4 \pm 423.16$ & $\begin{array}{l}\text { AD vs OSA, } \mathrm{p}<0.01 \\
\text { AD vs Controls, } \mathrm{p}<0.01 \\
\text { OSA vs Controls, } \mathrm{p}<0.05\end{array}$ \\
\hline $\mathrm{A} \beta 42 / \mathrm{A} \beta 40$ & $0.09 \pm 0.04$ & $0.03 \pm 0.03$ & $0.11 \pm 0.04$ & $\begin{array}{l}\text { AD vs } O S A, p<0.01 \\
\text { AD vs Controls, } \mathrm{p}<0.01 \\
\text { OSA vs Controls, } \mathrm{p}<0.05\end{array}$ \\
\hline T-tau $(p g / m L)$ & $205.35 \pm 98.59$ & $1059.7 \pm 598.95$ & $176.2 \pm 89.27$ & $\begin{array}{l}\text { AD vs } O S A, p<0.01 \\
A D \text { vs Controls, } p<0.01\end{array}$ \\
\hline P-tau $(p g / m L)$ & $39.1 \pm 14.88$ & $136.2 \pm 57.67$ & $38.3 \pm 6.84$ & $\begin{array}{l}\text { AD vs OSA, } p<0.01 \\
\text { AD vs Controls, } p<0.01\end{array}$ \\
\hline T-Tau/A $\beta 42$ & $0.42 \pm 0.38$ & $3.21 \pm 2.05$ & $0.20 \pm 0.10$ & $\begin{array}{l}\text { OSA vs Controls, } \mathrm{p}<0.05 \\
\mathrm{AD} \text { vs Controls, } \mathrm{p}<0.01 \\
\text { AD vs } \mathrm{OSA}, \mathrm{p}<0.01\end{array}$ \\
\hline Orexin $(p g / m L)$ & $604 \pm 95.99$ & $537.5 \pm 59.25$ & $486.25 \pm 88.54$ & $\begin{array}{l}\text { OSA vs Controls, } \mathrm{p}<0.05 \\
\mathrm{AD} \text { vs Controls, } \mathrm{p}<0.05 \\
\text { OSA vs } \mathrm{AD}, \mathrm{p}<0.05\end{array}$ \\
\hline
\end{tabular}

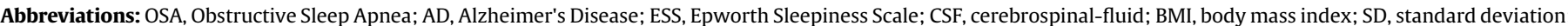
MMSE; Mini Mental State Examination; A $\beta 40, \beta$-amyloid40; A $\beta 42, \beta$-amyloid42; T-tau, total tau; P-Tau, phosphorylated tau; NS, not significant.

Table 2

PSG data of OSA, AD, and Control groups.

\begin{tabular}{|c|c|c|c|c|}
\hline PSG parameters & OSA $(n=20)$ mean $[S D]$ & $\mathrm{AD}(\mathrm{n}=20)$ mean $[\mathrm{SD}]$ & Controls $(\mathrm{n}=15)$ mean $[S D]$ & P Value \\
\hline TST (min) & $335.2[84.36]$ & 287.59 [61.73] & $298.25[67.37]$ & $\begin{array}{l}\text { OSA }>\text { Controls, }<0.01 \\
\mathrm{OSA}>\mathrm{AD},<0.01 \\
\mathrm{AD}<\text { controls, }<0.05\end{array}$ \\
\hline SE (\%) & $69.58[14.4]$ & $68.47[8.57]$ & $88.22[4.63]$ & $\begin{array}{l}\text { OSA }<\text { Controls, }<0.01 \\
\mathrm{AD}<\text { Controls, }<0.01\end{array}$ \\
\hline LREM (min) & $175.37[97.22]$ & 152.9 [6938] & $87.13[26.51]$ & $\begin{array}{l}\text { OSA }>\text { Controls, }<0.01 \\
\mathrm{AD}>\text { Controls, }<0.01\end{array}$ \\
\hline WASO (min) & $146.41[76.09]$ & $128.92[47.25]$ & $36.76[17.3]$ & $\begin{array}{l}\text { OSA }>\text { Controls, }<0.01 \\
\text { AD }>\text { Controls, }<0.01\end{array}$ \\
\hline $\mathrm{N} 1$ (min) & $58.42[44.16]$ & $69.76[51.62]$ & $15.12[4.97]$ & $\begin{array}{l}\text { OSA }>\text { Controls, }<0.01 \\
\mathrm{AD}>\text { Controls, }<0.01\end{array}$ \\
\hline $\mathrm{N} 2(\mathrm{~min})$ & $180.3[58.43]$ & $181.17[42.74]$ & $129.11[6.73]$ & $\begin{array}{l}\text { OSA }>\text { Controls, }<0.01 \\
\mathrm{AD}>\text { Controls, }<0.01\end{array}$ \\
\hline N3 (min) & $55.97[35.96]$ & $28.66[19.36]$ & $96.16[40.90]$ & $\begin{array}{l}\text { OSA }<\text { Controls, }<0.01 \\
\mathrm{AD}<\text { Controls, }<0.01 \\
\mathrm{AD}<\text { OSA, }<0.05\end{array}$ \\
\hline REM (min) & $40.5[20.69]$ & 35.15 [19.12] & $75.7[14.19]$ & $\begin{array}{l}\text { OSA }<\text { Controls, }<0.01 \\
\mathrm{AD}<\text { Controls, }<0.01\end{array}$ \\
\hline AHI & $33.7[19.01]$ & $9.5[4.17]$ & $7.3[2.16]$ & $\begin{array}{l}\text { OSA }>\text { Controls, }<0.01 \\
\text { OSA }>\text { AD, }<0.01\end{array}$ \\
\hline
\end{tabular}

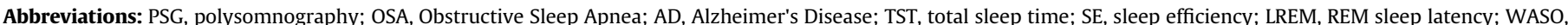

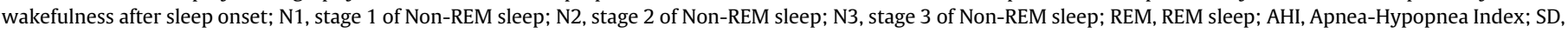
standard deviation.

CSF orexin and $A \beta_{42}$ levels did not correlate either in the OSA or in the $\mathrm{AD}$ group. Finally, we performed a multiple regression analysis in the OSA group documenting the significant interplay between CSF orexin levels and both AHI and WASO $(\beta=0.83$, $\mathrm{p}<0.01$ and $\beta=0.66, \mathrm{p}<0.05)$ and between $A \beta_{42} / \mathrm{A} \beta_{40}$ ratio and both $\mathrm{AHI}$ and $\mathrm{SE}(\beta=-0.63, \mathrm{p}<0.001$ and $\beta=0.46, \mathrm{p}<0.01)$.

\section{Discussion}

Brain beta-amyloid metabolism pathologically changes several years before the appearance of clinical symptoms of $A D$, which is the most frequent neurodegenerative disorder $[2] . \mathrm{AD}$ is neuropathologically featured by the accumulation of extracellular amyloid plaques of $A \beta_{42}$ aggregated peptides, and intracellular neurofibrillary tangles of tau proteins, both contributing to synaptic and neuronal loss $[2,31]$. Therefore, $A \beta_{42}$, t-tau and $p$-tau proteins have been considered the established CSF biomarkers supporting $A D$ diagnosis $[13,31]$. Furthermore, the ratios between t-tau and $A \beta_{42}$ and between $A \beta_{42}$ and $A \beta_{40}$ CSF levels actually represent further biomarkers for AD diagnosis [14,29].

In the present study, we documented the reduction of both $A \beta_{42}$ and $A \beta_{40}$ CSF levels in patients affected by moderate to severe OSA, as a possible expression of cerebral $\beta$-amyloid metabolism dysregulation. Moreover, OSA patients showed the alteration of the 


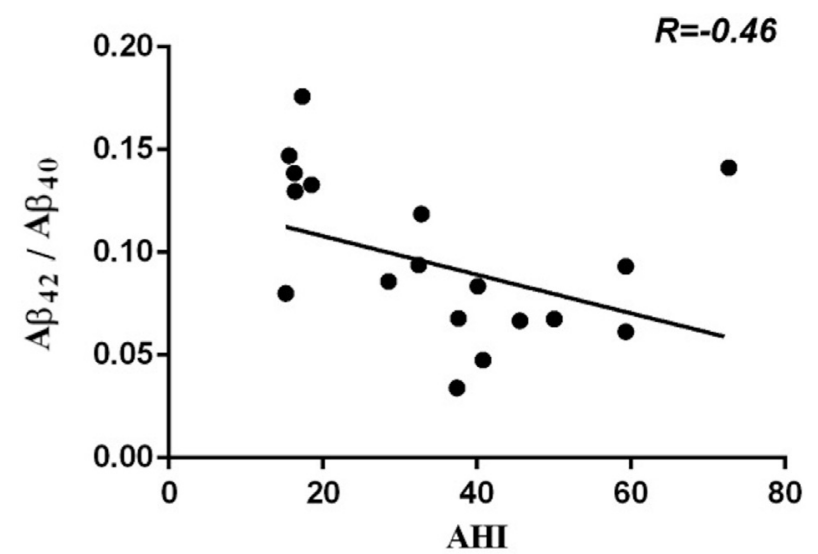

Fig. 1. Negative significant correlation between $A \beta_{42} / A \beta_{40}$ ratio and $A H I$ in the OSA group.

ratios indicative of $A D$ pathology $\left(A \beta_{42} / A \beta_{40}\right.$ and t-tau/A $\left.\beta_{42}\right)$. Finally, we documented that OSA patients, similarly to AD patients, not only showed the increase of CSF orexin concentrations but also the alteration of sleep architecture with the reduction of both REM sleep and SE and the increase of WASO and N2.

It is currently under debate whether OSA may promote $A D$ pathology $[9,34]$. Inflammation, sleep fragmentation, glymphatic dysfunction, and IH have been postulated as putative mechanisms triggering the $\mathrm{AD}$ neurodegenerative processes [1,4,6,35-38]. All these mechanisms are present in OSA patients, who show an increased risk for dementia, and in particular $A D$ and vascular dementia [9]. Accordingly, both the reduction of CSF A $\beta_{42}$ levels and the increase of brain $\beta$-amyloid plaques deposition have been already documented in OSA patients $[4,8]$. In this study, we have confirmed that OSA patients show the significant reduction of CSF $A \beta_{42}$ levels. In addition, we demonstrated that OSA patients also showed the decrease of CSF A $\beta_{40}$ levels. Since both the main isoforms of $\beta$-amyloid were reduced in the CSF of OSA patients, the pathological modification of cerebral $\beta$-amyloid dynamics can be supposed. However, the candidate mechanisms at the basis of this dysregulation can be exclusively hypothesized. Following a previous hypothesis drawn from observations achieved in animal models studies, $\mathrm{IH}$ has been supposed altering the brain metabolism of $\beta$-amyloid [36], and sleep fragmentation complemented with glymphatic dysfunction has been associated with brain interstitial $\beta$-amyloid deposition and CSF $\beta$-amyloid levels alteration $[1,35,37]$. Therefore, sleep impairment and IH may explain the alteration of CSF biomarkers in OSA patients, which are similar to that of $A D$ (reduced $A \beta_{42}$ CSF levels, increased t-tau/A $\beta_{42}$ ratio), thus further supporting the hypothesis that OSA may start AD pathology. Moreover, we documented a quite high percentage of apoE-e4 carriers (40\%) in OSA patients (which is similar to that of controls) and not different from AD patients (60\%). This finding may be responsible for the alteration of CSF biomarkers documented in OSA patients, also considering that controls present normal CSF biomarkers levels despite having a similar prevalence of apoE-e4 carriers. OSA patients also showed reduced CSF $A \beta_{40}$ levels, which is not a typical finding in $A D$, since CSF $A \beta_{40}$ levels in AD patients have been reported as similar to controls [3]. Thus, the changes seen in $\beta$-amyloid metabolism in OSA patients may appear quite different than the ones presented by $A D$ patients, and may not be directed to the $\beta$-amyloid aggregationprone isoforms. The more suggestive hypothesis can be related to the reduced production of $\beta$-amyloid isoforms, although this supposition has to be completely proved.

The novelty of this study lies in the increase of CSF orexin levels detected in moderate-severe OSA patients. Orexin is a wake- promoting neurotransmitter and its pathological reduction has been associated with narcolepsy, a neurological disorder characterized by excessive daytime sleepiness [33]. In past years, several studies assessed plasma orexin levels in OSA patients with controversial results, and only a single uncontrolled study measured normal CSF orexin levels in a small group of patients affected predominantly by mild OSA and reporting daytime sleepiness [21-23]. Furthermore, we confirmed the previous observation that AD patients show higher CSF orexin levels compared to controls. Increased orexinergic tone has been already demonstrated in $\mathrm{AD}$ patients from the early stages of the disease, although a post-mortem study documented the reduction of orexinergic neurons and CSF orexin levels [17,19,38,39]. On one hand, the higher CSF levels of orexin in AD patients has been related to sleep impairment and cognitive decline $[17,19,38]$. Conversely, the higher CSF orexin levels found in moderate-severe OSA patients is a novel finding, and the pathophysiological basis can be speculated. Since OSA patients present a significant alteration of sleep architecture, characterized by increased WASO and several arousals, we may hypothesize that the increase of CSF orexin levels may be a secondary effect of sleep dysregulation and increased nocturnal wakefulness. Accordingly, orexin is a neurotransmitter which promotes wakefulness and suppresses sleep [40]. Moreover, OSA patients frequently present with daytime sleepiness, and therefore the higher CSF orexin levels present in OSA patients may be also the possible expression of a compensatory mechanism counteracting daytime somnolence. Nevertheless, we did not found significant correlations between CSF orexin levels and ESS scores. In keeping with this observation, sleepiness is a complex phenomenon with different neurobiological causes, not only related to orexinergic neurotransmission. Since it has been proved that REM sleep suppression increases CSF orexin levels [41,42], another possible explanation may be related to the reduced REM sleep present in OSA patients. However, we did not document associations between CSF orexin levels and sleep stages changes in OSA patients, but we documented the relation between higher CSF orexin levels and the increase of both AHI and WASO, thus supporting that nocturnal wakefulness and apnea-hypopnea events may be related to the higher orexinergic tone found in OSA patients. Consistently, orexin neurons are the most $\mathrm{CO} 2 / \mathrm{H}+$ sensitive neurons in the brain and they are activated by $\mathrm{CO} 2$ in vivo $[43,44]$. Concurrently, the weak reduction of $A \beta_{42} / A \beta_{40}$ ratio present in OSA patients related to the increased AHI and the reduced SE. Hence, it is also reasonable that $\mathrm{IH}$ and hypercapnia may not only activate orexin neurons, thus increasing CSF orexin levels, but also alter $\beta$ amyloid metabolism, thus reducing the $A \beta_{42} / A \beta_{40}$ ratio.

Considering AD patients, CSF orexin levels significantly correlated with sleep architecture changes (as already proved) [17,19] and concentrations of several CSF biomarkers, such as $A \beta_{40}$, t-tau and $\mathrm{p}$-tau; in addition, both ratios considered for $\mathrm{AD}$ diagnosis (ttau/A $\beta_{42}$ and $\left.A \beta_{42} / A \beta_{40}\right)$ correlated with CSF orexin levels. This finding further looks into the previously reported hypothesis that orexin may modulate not only CSF A $\beta_{42}$ levels but also the other CSF AD biomarkers [17,19,37,45].

OSA actually represents an increasing health problem in the adult population, and it is associated not only with several metabolic and cardio-vascular injuries, but also with the occurrence of cognitive decline and brain functional and morphological changes $[46,47]$. Moreover, OSA may negatively modulate cerebral $\beta$-amyloid metabolism and increases CSF orexin concentrations, thus possibly representing a risk factor for early $\mathrm{AD}$ neuropathological modifications. Accordingly, increasing literature suggests that OSA is not only a frequent diagnosis in $\mathrm{AD}$ patients, but also may cause $\mathrm{AD}$ [48]. Following this hypothesis, the reduction of CSF $A \beta_{42}$ levels and the increase of CSF orexin concentrations found in OSA patients 
may be explained by the fact that OSA may induce preclinical AD biomarkers changes, such those possibly documented in the early stages of $\mathrm{AD}$. Yet, the other biomarkers changes documented in OSA patients and not directed at AD pathology invite further research to better address this hypothesis.

In conclusion, our data raise the scientific interest on this topic, which is directed at understanding the effects of sleep disorders with widespread prevalence, such as OSA, and on brain health. Thus, we propose future research and international collaborations and networks, which have to be organized in order to produce further results including larger populations of patients.

\section{Conflict of interest}

This was not an industry-supported study. The authors have no financial disclosures or conflicts of interest.

The ICMJE Uniform Disclosure Form for Potential Conflicts of Interest associated with this article can be viewed by clicking on the following link: https://doi.org/10.1016/j.sleep.2019.01.003.

\section{References}

[1] Xie L, Kang H, Xu Q et al. Sleep drives metabolite clearance from the adult brain. Science 2013 Oct 18:342(6156):373-7.

[2] Yaari R, Corey-Bloom J. Alzheimer's disease. Semin Neurol 2007;27(1):32-41.

[3] Haass C, Selkoe DJ. Soluble protein oligomers in neurodegeneration: lessons from the Alzheimer's amyloid beta-peptide. Nat Rev Mol Cell Biol 2007;8(2): $101-12$.

[4] Liguori C, Mercuri NB, Izzi F, et al. Obstructive sleep apnea is associated with early but possibly modifiable Alzheimer's disease biomarkers changes. Sleep 2017;40(5).

[5] Ju Y-ES, Finn MB, Sutphen CL, et al. Obstructive sleep apnea decreases central nervous system-derived proteins in the cerebrospinal fluid. Ann Neurol 2016:80(1):154-9.

[6] Liguori C, Chiaravalloti A, Izzi F, et al. Sleep apnoeas may represent a reversible risk factor for amyloid- $\beta$ pathology. Brain J. Neurol. 2017;140(12):e75.

[7] Sharma RA, Varga AW, Bubu OM, et al. Obstructive sleep apnea severity affects amyloid burden in cognitively normal elderly. A longitudinal study. Am J Respir Crit Care Med 2018;197(7):933-43.

[8] Yun C-H, Lee H-Y, Lee SK, et al. Amyloid burden in obstructive sleep apnea. J. Alzheimers Dis. JAD 2017;59(1):21-9.

[9] Shi L, Chen S-J, Ma M-Y, et al. Sleep disturbances increase the risk of dementia: a systematic review and meta-analysis. Sleep Med Rev. 2018 Aug;40:4-16.

[10] Ryan CM, Bradley TD. Pathogenesis of obstructive sleep apnea. J. Appl. Physiol. Bethesda Md 1985 2005:99(6):2440-50.

[11] Sateia MJ. International classification of sleep disorders-third edition: highlights and modifications. Chest 2014;146(5):1387-94.

[12] Daulatzai MA. Evidence of neurodegeneration in obstructive sleep apnea: relationship between obstructive sleep apnea and cognitive dysfunction in the elderly. J Neurosci Res 2015;93(12):1778-94.

[13] McKhann GM. Changing concepts of Alzheimer disease. J Am Med Assoc 2011:305(23):2458-9.

[14] Baldeiras I, Santana I, Leitão MJ, et al. Addition of the A $\beta 42 / 40$ ratio to the cerebrospinal fluid biomarker profile increases the predictive value for underlying Alzheimer's disease dementia in mild cognitive impairment. Alzheimer's Res Ther 2018;10(1):33.

[15] Saper CB, Scammell TE, Lu J. Hypothalamic regulation of sleep and circadian rhythms. Nature 2005;437(7063):1257-63.

[16] Fronczek R, van Geest S, Frölich M, et al. Hypocretin (orexin) loss in Alzheimer's disease. Neurobiol Aging 2012;33(8):1642-50.

[17] Liguori C, Romigi A, Nuccetelli M, et al. Orexinergic system dysregulation, sleep impairment, and cognitive decline in Alzheimer disease. JAMA Neurol 2014;71(12):1498-505.

[18] Slats D, Claassen JAHR, Lammers GJ, et al. Association between hypocretin-1 and amyloid- $\beta 42$ cerebrospinal fluid levels in Alzheimer's disease and healthy controls. Curr Alzheimer Res 2012:9(10):1119-25.

[19] Liguori C, Nuccetelli M, Izzi F, et al. Rapid eye movement sleep disruption and sleep fragmentation are associated with increased orexin-A cerebrospinal- fluid levels in mild cognitive impairment due to Alzheimer's disease. Neurobiol Aging 2016:40:120-6.

[20] Roh JH, Jiang H, Finn MB, et al. Potential role of orexin and sleep modulation in the pathogenesis of Alzheimer's disease. J Exp Med 2014 Dec 15;211(13): 2487-96.

[21] Busquets X, Barbe F, Barcelo A, et al. Decreased plasma levels of orexin-A in sleep apnea. Respiration 2004;71:575-9.

[22] Igarashi N, Tatsumi K, Nakamura A, et al. Plasma orexin-A levels in obstructive sleep apnea-hypopnea syndrome. Chest 2003:124:1381-5.

[23] Kanbayashi T, Inoue Y, Kawanishi K, et al. CSF hypocretin measures in patients with obstructive sleep apnea. J Sleep Res 2003;12(4):339-41.

[24] Iber C, Ancoli-Israel S, Chesson Jr AL, et al. The AASM manual for the scoring of sleep and associated events: rules, terminology, and technical specifications. 1st ed. Westchester, IL: American Academy of Sleep Medicine; 2007.

[25] Heinzer R, Vat S, Marques-Vidal P, et al. Prevalence of sleep-disordered breathing in the general population: the HypnoLaus study. Lancet Respir Med 2015 Apr;3(4):310-8.

[26] Gehrman PR, Martin JL, Shochat T, et al. Sleep-disordered breathing and agitation in institutionalized adults with Alzheimer disease. Am J Geriat Psychiatry 2003 Jul-Aug;11(4):426-33.

[27] Vignatelli L, Plazzi G, Barbato A, et al. Italian version of the Epworth sleepiness scale: external validity. Neurol. Sci. Off. J. Ital. Neurol. Soc. Ital. Soc. Clin. Neurophysiol. 2003;23(6):295-300.

[28] Johns MW. A new method for measuring daytime sleepiness: the Epworth sleepiness scale. Sleep 1991;14(6):540-5.

[29] Duits FH, Teunissen CE, Bouwman FH, et al. The cerebrospinal fluid "Alzheimer profile": easily said, but what does it mean? Alzheimers Dement. J. Alzheimers Assoc. 2014;10(6):713-723.e2.

[30] Mulder C, Verwey NA, van der Flier WM, et al. Amyloid-beta(1-42), total tau, and phosphorylated tau as cerebrospinal fluid biomarkers for the diagnosis of Alzheimer disease. Clin Chem 2010;56(2):248-53.

[31] Fagan AM, Mintun MA, Mach RH, et al. Inverse relation between in vivo amyloid imaging load and cerebrospinal fluid Abeta42 in humans. Ann Neurol 2006:59(3):512-9.

[32] Liguori C, Romigi A, Mercuri NB, et al. Cerebrospinal-fluid orexin levels and daytime somnolence in frontotemporal dementia. J Neurol 2014;261(9): 1832-6.

[33] Ripley B, Overeem S, Fujiki N, et al. CSF hypocretin/orexin levels in narcolepsy and other neurological conditions. Neurology 2001;57(12):2253-8.

[34] Liguori C, Placidi F. Is it time to consider obstructive sleep apnea syndrome a risk factor for Alzheimer's disease? Am J Respir Crit Care Med 2018;197(7): 855-6.

[35] Ju Y-ES, McLeland JS, Toedebusch CD, et al. Sleep quality and preclinical Alzheimer disease. JAMA Neurol 2013;70(5):587-93.

[36] Shiota S, Takekawa H, Matsumoto S-E, et al. Chronic intermittent hypoxia reoxygenation facilitate amyloid- $\beta$ generation in mice. J. Alzheimers Dis. JAD 2013;37(2):325-33.

[37] Ooms S, Overeem S, Besse K, et al. Effect of 1 night of total sleep deprivation on cerebrospinal fluid $\beta$-amyloid 42 in healthy middle-aged men: a randomized clinical trial. JAMA Neurol 2014;71(8):971-7.

38] Gabelle A, Jaussent I, Hirtz C, et al. Cerebrospinal fluid levels of orexin-A and histamine, and sleep profile within the Alzheimer process. Neurobiol Aging 2017;53:59-66

[39] Fronczek R, van Geest S, Frölich M, et al. Hypocretin (orexin) loss in Alzheimer's disease. Neurobiol Aging 2012 Aug:33(8):1642-50.

[40] Hagan JJ, Leslie RA, Patel S, et al. Orexin A activates locus coeruleus cell firing and increases arousal in the rat. Proc Natl Acad Sci USA 1999;96(19):10911-6.

41] Eggermann E, Bayer L, Serafin M, et al. The wake-promoting hypocretinorexin neurons are in an intrinsic state of membrane depolarization. J. Neurosci. Off. J. Soc. Neurosci. 2003;23(5):1557-62.

[42] Pedrazzoli M, D'Almeida V, Martins PJF, et al. Increased hypocretin-1 levels in cerebrospinal fluid after REM sleep deprivation. Brain Res 2004:995(1):1-6.

[43] William RH, Jensen LT, Verkhratsky A, et al. Control of hypothalamic orexin neurons by acid and $\mathrm{CO}_{2}$. Proc Natl Acad Sci USA 2007;104:10685-90.

[44] Sunanaga J, Deng BS, Zhang W, et al. $\mathrm{CO}_{2}$ activates orexin-containing neurons in mice. Respir Physiol Neurobiol 2009;166:184-6.

[45] Osorio RS, Ducca EL, Wohlleber ME, et al. Orexin-a is associated with increases in cerebrospinal fluid phosphorylated-tau in cognitively normal elderly subjects. Sleep 2016:39(6):1253-60.

[46] Torelli F, Moscufo N, Garreffa G, et al. Cognitive profile and brain morphological changes in obstructive sleep apnea. Neuroimage 2011;54(2):787-93.

[47] Canessa N, Ferini-Strambi L. Sleep-disordered breathing and cognitive decline in older adults. J Am Med Assoc 2011:306(6):654-5.

[48] Osorio RS, Gumb T, Pirraglia E, et al. Sleep-disordered breathing advances cognitive decline in the elderly. Neurology 2015;84(19):1964-71. 